\title{
A wing growth organizer in a hemimetabolous insect suggests wing origin
}

Takahiro Ohde ${ }^{1,2,3^{*}}$, Taro Mito ${ }^{4}$ and Teruyuki Niimi2,3

1Department of Applied Biosciences, Graduate School of Agriculture, Kyoto University, Kyoto, Japan

2Division of Evolutionary Developmental Biology, National Institute for Basic Biology, Okazaki, Japan

3Department of Basic Biology, School of Life Science, SOKENDAI (The Graduate University for Advanced Studies), Okazaki, Japan

4Division of Bioscience and Bioindustry, Graduate School of Technology, Industrial and Social Sciences, Tokushima University, Tokushima, Japan

*Correspondence to: ohde.takahiro.4n@kyoto-u.ac.jp 


\section{ABSTRACT}

2 The origin and evolution of insect wings remain enigmatic after a century-long discussion.

3 Molecular dissection of wing development in hemimetabolous insects, in which the first

4 functional wings evolved, is key to understand genetic changes required for wing evolution.

5 We investigated Drosophila wing marker genes in the cricket, Gryllus bimaculatus, and found

6 apterous and vestigial show critical functions in nymphal tergal identity and margin formation,

7 respectively. We further demonstrate that margin cells in the lateral-anterior tergal region

8 constitute a growth organizer of wing blades. Transcriptome and RNAi analyses unveiled that

9 Wnt, Fat-Dachsous, and Hippo pathways are involved in disproportional growth of Gryllus

10 wings. Our data collectively support the idea that tergal margin cells of a wingless ancestor

11 gave rise to the body wall extension required for evolution of the first powered flight.

12 


\section{INTRODUCTION}

14 Insects were the first animal group to achieve powered flight. The significance of this development for successful radiation of this animal group is unarguable, yet the evolutionary history of the wing is still unclear after discussion over a century. The dispute has focused primarily on two concepts of wing origin: (1) extension of the dorsal body wall (e.g. Crampton, 1916; Snodgrass, 1935), and (2) modification of a pre-existing structure related to a ventral appendage (e.g. Wigglesworth, 1978; Kukalova-Peck, 1983). These concepts link the wing to anatomically distinct body parts of the insect ground plan, tergum and pleuron, respectively, and evolutionary history of the wing is separately illustrated for each hypothesis (ClarkHachtel and Tomoyasu, 2016). A dual origin hypothesis reconciles these classical ideas and proposes that both tergal and pleural elements fused to form the first functional wings (Rasnitsyn, 1981; Clark-Hachtel and Tomoyasu, 2016). This idea has gained support in recent developmental and paleontological studies. Still, the hypothesis embraces a spectrum of interpretations on the degree of contribution from each element (Linz and Tomoyasu, 2018). Whereas understanding of the genetic regulation of wing development has accumulated in Drosophila, this knowledge is largely lacking in other insects, particularly outside the Holometabola. Holometabolous larvae undergo dynamic remodeling of the entire body organization during pupation to form the adult insect. The evolution of holometabolous development split the life cycle into two modules and facilitated modification of ancestral hemimetablous development (Truman \& Riddiford, 2019). This modified mode of development makes it less suitable to directly compare it with the development of apterygote ancestors for understanding wing evolution. nymphs and adults. Body parts that change substantially in size and pattern during the 
37 transition include genitalia and wings. In the modern hemimetabolous insects, wings are not

38 visible in early nymph stages, and evaginated wing primordia, called wing pads,

39 progressively expand and become patterned at each molt. The first winged insects

40 developed obviously in hemimetabolous species, and dissecting hemimetabolous wing

41 development at the molecular level is essential for deducing genetic changes responsible for

42 the rise of wings from an ancestral wingless body plan. In the two-spotted cricket, Gryllus

43 bimaculatus, wings extend from lateral areas of nymphal thoracic terga (Mashimo and

44 Machida, 2017), but genetic mechanisms underlying this process are not known. 


\section{RESULTS}

\section{$w g, a p A B$ and $v g$ mRNAs expression in developing thoracic terga in Gryllus embryos}

Given their central roles in the wing development of Drosophila melanogaster, vestigial ( $\mathrm{vg})$, apterous (ap) and wingless ( $w g$ ) were selected as wing marker genes for finding structures homologous to wings in other insects and crustaceans (Averof and Cohen, 1997; Niwa et al., 2010; Ohde et al., 2013; Clark-Hachtel et al., 2013; Hu et al., 2019; Clark-Hachtel and Tomoyasu, 2020). vg in Drosophila embryos is the earliest marker of wing and haltere imaginal disc cells, although its role in determination of imaginal disc fate is unclear (Williams et al., 1991). $v g$ is required for the selective proliferation and identity formation of the prospective wing region of larval imaginal discs (Williams et al., 1991; Kim et al., 1996). In late second instar larvae, Ap in the dorsal compartment of the wing disc induces both $v g$ boundary enhancer ( $v g \mathrm{BE})$ activity and wg expression at the dorsoventral (DV) boundary via the Notch pathway (Williams et al., 1994; Diaz-Benjumea and Cohen, 1995; Kim et al., 1995). Subsequently, Wg from DV boundary cells, together with Decapentaplegic (Dpp) from anteroposterior (AP) boundary cells, activates $v g$ quadrant enhancer ( $v g \mathrm{QE})$ to expand the wing compartment in the wing disc (Kim et al., 1996; Zecca and Struhl, 2007).

We initially examined expression patterns of $w g$, $a p A B$, and $v g$ in stages 6,7 , and 9 embryos of Gryllus. Tergal and pleural regions are subdivided during these stages (Fig. 1A, Mashimo and Machida, 2017). In the thoracic tergal region, spots of wg mRNA expression appear on the posterior side in early stage 7 embryos and become more prominent in late-stage 7 (Fig. 1B, C). Additional $w g$ expression in lateral to posterior tergal edges is detected in stage 9 (Fig. 1D). wg is expressed in stripe patterns at the AP boundary of legs in the pleural region, as previously reported (Fig. 1B-D; Niwa et al., 2000). Two ap orthologs in the Gryllus genome were classified to $\mathrm{ApA}$ and $\mathrm{ApB}$ from six amino acid residues unique to each group 
in the homeodomain (Fig. S1). However, partial sequences we obtained were insufficient to separately analyze in situ expression of each gene (Fig. S1). We detected apAB signals in thoracic terga of stage 6 embryos that were maintained to stage 9 (Fig. 1E-G). apAB expression covers a broad area of thoracic terga, yet marginal cells lack expression. $a p A B$ expression is also detected in the thoracic pleural regions in addition to tergal cells. $a p A B$ is expressed in a U-shape pattern in stage 6, becomes separated into two areas in late stage 7, and forms connected areas, reshaping the $U$ in stage 9 (Fig. 1E-G). Clear vg signals were first detectable at stage 9, in contrast to $w g$ and $a p A B(\mathbf{F i g} . \mathbf{1 H}-\mathbf{J})$. Expression localized to tergal margins and two pleural spots (Fig. 1J). Marginal expression patterns of $w g$ and $v g$ in stage 9 embryos are both localized to the lateral to posterior regions of meso- and metathorax (T2 and T3), and in the anterior region of prothorax (T1) (Fig. 1D, G, J, K). $v g$ is an embryonic marker of wing disc cells in Drosophila (Williams et al., 1991). The wing disc includes a primordium of the wing and notum and pleural tissues. The formation of this compact imaginal disc is characteristic only in higher Diptera (Nijhout, 1994), and the fate of $v g$-expressing embryonic cells in insects other than Drosophila is unknown. In Gryllus, the evidence is limited to mRNA expression patterns with in situ hybridization in late embryos due to outer cuticle formation that causes strong non-specific staining (Niwa et al., 2000). We generated a $v g$ reporter line by knocking-in the EGFP cassette at the upstream side of the $v g$ coding sequence (Fig. S2A-C). This reporter line (vg5'GFP) mimics $v g$ mRNA expression in both tergal and pleural regions at stage 9 (Fig. 1K). During late embryonic development, the EGFP signal pattern in the pleural region is transformed from two spots to several cylindrical shapes (stage 12-), but the tergal region is maintained without significant change from earlier stages (Fig. 1K-M, Fig. S2C-Q). vg reporter signal in nymphs appears at the margins of wing pads while strong signaling disappears in the posterior tergal margin 
regions (Fig. 1N, N'). A signal was also detected in thoracic dorsoventral muscles (Fig. 10, thoracic musculature including dorsal longitudinal and dorsoventral and pleural muscles that are indirect- and direct flight muscles, respectively (Fig. 1Q, Q', Fig. S3; Furukawa et al., 1983).

100

\section{$a p A B$ and $v g$ function in nymphal thoracic terga and adult wing formation}

We investigated the function of wing marker genes in body plan formation of Gryllus by generating CRISPR/Cas9-mediated mosaic knockouts for each gene. A loss of wg function likely causes no effects due to functional redundancy (Miyawaki et al., 2004), and we focused on $v g$ and $a p A B$ functions. We designed a sgRNA targeting EGFP as a negative control (Fig.

2A-E; Table S1). Consistent with the expression pattern during embryogenesis, vg sgRNA/Cas9 injected individuals ( $\left.v g^{C R I S P R}\right)$ exhibited a lack of tergal margins in both wingless (T1) and wing-bearing (T2 and T3) segments of first instar nymphs (Fig. 2F-H). Further, individuals without thoracic tergal margins displayed severely reduced wings in adults (Fig. 2I, J). Characteristic patterns in thoracic terga in apABCRISPR individuals, such as color, shape, and hairs were lost (Fig. 2K-M). Affected tergal areas lost their normal smooth surface, replaced by the rough surface seen in intersegmental membranes of wildtype insects (Fig. $2 \mathrm{~N})$. Loss of black color was also induced in lateral-posterior regions of abdominal segments in $a p A B C R I S P R$ nymphs (Fig. 2K). These nymphs did not survive to adults. 
$118 v g$ CRISPR suggests that the tergal margin region associated with $v g$ expression during 119 embryogenesis is required for wing growth. During post-embryonic development, lateral regions in T2 and T3 that give rise to wings show dramatic exponential growth, while the rest the same segment exhibits linear growth (Fig. S4). $v g$ expression and function in Gryllus embryos are displayed in lateral and posterior margin regions of T2 and T3, and this growth pattern suggests that the lateral region comprises the wing growth organizer. We ablated a part of the mesothoracic tergum in the third instar nymph to test this hypothesis and analyzed effects on adult wing size (Fig. 3A). Adult wing size is unaffected after posterior tergum ablation, while removal of lateral regions severely reduced wing size (Fig. 3B). This effect is more prominent when larger regions of the lateral tergum are ablated (Fig. 3B). A similar size reduction was found in wing articulation, although the effect is small (Fig. 3C). We independently ablated anterior and posterior regions to further identify the lateral region critical to wing formation (Fig. 3A). We found that removal of the anterior region results in almost complete loss of the wing blade, whereas removal of the posterior region has almost no effect on wing size (Fig. 3B, D-G). Ablation of the lateral-anterior (LA) region of a tergum is sufficient to cause the loss of almost the entire wing blade, but effects are less prominent on the size of the wing articulation (Fig. $\mathbf{3 C}$ ). Thus, the growth organizer of wing blades located in the LA region of terga.

A combination of transcriptome and RNAi analyses revealed that Wnt, Fat, and Hippo signaling are required for post-embryonic wing growth

We next compared transcriptomes between lateral and central parts of terga (Fig. 4A) to identify genes that regulate the growth of lateral tergal regions in T2 and T3. The steroid hormone 20-hydroxyecdysone (20E) promotes wing growth by increasing both cell number 
142 and cell size in lepidopteran insects (Nijhout \& Grunert, 2002; Nijhout et al., 2018). We thus 143 examined the expression level of two ecdysone responsive genes, $E 74$ and $E 75$, as proxies 144 for hemolymph ecdysone titer to determine the timing of tissue sampling for RNA-seq analysis (Ashburner \& Richards, 1976; Li et al., 2003). E74 and E75 in thoracic segments showed expression peaks at 3- and 2.5-days post ecdysis to third instar (DPE), respectively (Fig. S5). We selected 3 DPE as a growing stage since total RNA yield is highest among third instar nymphs, suggesting active transcription and protein synthesis (Fig. S5). We obtained raw reads from lateral and central regions of both T2 and T3 in 0 DPE and 3 DPE with a sufficient quality (Q30 >95.87\%; Fig. S6A). We assembled a transcriptome from these reads with embryonic reads (BUSCO complete orthologs 95.6\%; Fig. S6B, C), and quantified expression level of each transcript. Transcriptomes display greater similarity within time point than within regions (Fig. S7A). We analyzed differentially expressed genes (DEGs) between regions (Lateral versus Central) and between time points (0 DPE versus 3 DPE). We detected more DEGs between time points than between regions, consistent with the above findings (Fig. S7B). We presumed 3 DPE as a growing stage, and transcripts highly expressed during this time should be enriched with keywords such as "Developmental protein" and "Cell division", implying that genes involved in the growth of the lateral region were successfully identified (Fig. S7C). DEGs in the lateral region were enriched with the keywords such as "DNA replication", "Cell cycle", and "Developmental protein", support our hypothesis that the lateral terga comprise the growth organizer in T2 and T3 (Fig. S7C). We then proceeded to develop a list of transcripts for RNAi-mediated functional screening. We focused on transcripts annotated as signaling molecules or transcription factors among DEGs more highly expressed in the lateral region than the central region to identify upstream wing growth regulators (Fig. 4C). Notably, we found that genes involved in Wnt (Wnt ligands, 

pathways generate a feed-forward (FF) circuit that plays a central role in the expansion of the wing compartment in Drosophila (Fig. 4B, C; Zecca \& Struhl, 2010). In contrast, components of BMP signaling, another well-characterized signaling pathway that plays a pivotal role in the Drosophila wing disc growth and patterning, was not identified as a DEG between regions (Fig. S8; Hamaratoglu, et al., 2014).

We selected 33 genes, including components of Wnt, Ft-Ds, and Hippo pathways, as candidate growth regulators and performed nymphal RNAi (nRNAi)-mediated functional screening to examine functions in Gryllus wing formation (Fig. 4C, Table S2). We found specific phenotypes for six transcripts: two ft transcripts, $d$, the T-box transcription factor optomotor-blind (omb), Epidermal growth factor receptor (Egfr), zinc finger homeodomain 2 (zfh2), and abrupt (ab). A BLAST-based comparison to the Drosophila official gene showed two Gryllus $f t$ transcripts that showed effects on the wing size after RNAi treatment derive from a single $f t$ locus (Fig. S9). Among six genes with specific phenotypes, depletion of $f t$ resulted in reduced wing size compared to control EGFP dsRNA injected crickets, suggesting a role of Ft-Ds pathways in the Gryllus wing growth. omb RNAi crickets exhibit irregular wing vein patterns around the AP boundary (Fig. S10A-D). RNAi treatment of $z$ fh2 caused high lethality, but surviving adults commonly showed disorganized vein patterns (Table S2; Fig. 
190

slightly smaller wings although this effect was not clear because of the modest impact (Fig. S10H). We targeted genes encoding effectors of Hippo pathway, scalloped (sd) and yorkie $(y k i)$, to further confirm the role of the Hippo pathway in wing growth. Depletion of $s d$ caused a severe reduction in wing size (Fig. $\mathbf{4 H}$; Table S3). We failed to analyze the effect of $y k i$ dsRNA injections of third instar nymphs on wing growth due to lethality, but injection of sixth instar nymphs severely reduced wing size (Fig. 4I; Table S3). In contrast, we found no noticeable effect on wing formation after a single knockdown of each Wnt pathway component (Table S2). This result may reflect functional redundancy of ligands and receptors reported in Gryllus and other species (Miyawaki et al., 2004).

We targeted intercellular components of the canonical Wnt signaling pathway disheveled (dsh) and armadillo (arm) to clarify the involvement of the Wnt pathway (Table S3). RNAi treatment for both genes resulted in severe reductions in wing size, showing the central role of the Wnt pathway in the Gryllus wing growth. Separate RNAi-treatment against $f t, y k i, s d$, dsh and arm resulted in the reduced size of both female ovipositor and antennae (Fig. S11A, B). $d$ RNAi crickets displayed curved and short ovipositors (Fig. S11C). Ovipositor and antenna size indicate disproportional growth during post-embryonic development, suggesting a shared role of Wnt/Ft-Ds/Hippo pathways in the growth regulation among disproportionally growing organs. 


\section{DISCUSSION}

\section{vg-dependent LA cells organize the wing growth in Gryllus}

211 In Gryllus, ap is expressed across a broad region of terga in stage 9, and $w g$ and $v g$ show

212 localized expression in thoracic tergal margin cells. Such a spatial gene expression in Gryllus

213 embryos is analogous to expression in Drosophila wing discs in late second instar larvae. In

214 Drosophila, ap induces $v g$ and $w g$ at the DV boundary to organize wing formation (Kim et al.,

215 1995). These three genes show no similar spatial distribution during Drosophila

216 embryogenesis. These results demonstrate the timing shift of patterning event from embryonic development in Gryllus to post-embryonic development in Drosophila (Fig. 5). This temporal difference in gene expression might be explained by a developmental modification to form holometabolous larvae. From examples in the development of CNS, legs and eyes, Truman \& Riddiford (2019) formulated the principle underlying the evolution of holometabolous larval forms: (1) arrest of ancestral embryonic development programs and (2) redirection of development to an adaptive holometabolous larval form. We ascribe the different timing of the analogous gene expression between Gryllus and Drosophila to a similar timing shift through the evolution from the hemimetabolous to holometabolous mode. The functional comparison of $a p$ and $v g$ between the Drosophila larval wing disc and the Gryllus embryos further supports the concept of a timing shift during evolution, and implicates an ancestral role of $v g$ in the formation of the wing organizer. Ap specifies and maintains dorsal cell fate in the Drosophila wing disc (Diaz-benjumea \& Cohen, 1993). We show ap function in the tergal identity formation in the Gryllus embryo, indicating an evolutionarily conserved function with different timing of expression between species. $v g$ repeatedly plays important roles in Drosophila wing development. First, $v g$ marks all wing disc-fated cells in embryos by stage 11 (Williams et al., 1991). Second, the priming enhancer induces low-level 
$v g$ expression across the entire wing disc. Such expression is required for the initiation of wing growth (Zecca \& Struhl, 2008). Third, vgBE-regulated $v g$ expression organizes feedforward growth in the wing region (Williams et al., 1994; Zecca \& Struhl, 2010). Fourth, vgQE integrates signals from multiple pathways to expand the wing region (Zecca, 2008; 2010). Analogous expression at the DV boundary between Drosophila and Gryllus suggests that the third function in organizing wing growth could derive from ancestral hemimetabolous development. Our functional analysis and ablation experiment in Gryllus consistently show the lack of a part of the $v g$-dependent tergal margin, the LA region, causes severe wing blade growth defects. In summary, $v g$ forms the thoracic tergal margins in the embryonic development, and the vg-dependent LA cells organize the wing growth in the postembryonic development.

\section{Evolutionarily conserved roles of $v g$ and $a p$ in flight muscle development}

$v g$-expressing cells at the tergal region are critical for the formation of the wing growth organizer, but it is the pleural region that appears to be involved in muscle development. Our detailed reporter analysis showed that the pleural population likely forms myoblasts that differentiate into nymphal dorsoventral muscles. Some nymphal muscles are used for flight in adult insects (Tiegs, 1954). We also detected consistently strong vg reporter signals in both indirect and direct flight muscles in T2 and T3 of adults, indicating a likely involvement of $v g$ in adult flight muscle development of Gryllus. This possibility is concordant with a previous report that $v g$ patterns indirect flight muscles, both dorsoventral and dorsal longitudinal, by repressing ap and cut. These latter genes specify direct flight muscles in 
development, although the contrasting expression pattern of $v g$ in adult direct flight muscles, that is present in Gryllus and absent in Drosophila (Sudarson et al., 2001), implies the modified regulation between species. extend tergal margins of the wingless ancestor

Large wing blades are fundamental to sufficient aerodynamic forces for powered flight. Hence, disproportionate growth of the lateral body wall that gives rise to the wing blade is an essential step for the evolution of functional flight; however, mechanisms underlying this step remain unclear. Each hypothesis for wing origin suggests different scenarios for wing evolution, yet all hypotheses appear to agree on a major contribution of the tergal margin region of the apterygote insect ancestor in the formation of the wing blade (Snodgrass, 1935; 2021; Tomoyasu, 2021). The location of a wing growth organizer at the lateral tergal region in Gryllus is concordant with this view. Distribution of $w g$, ap and $v g$ mRNAs along the DV axis in Gryllus is similar to the distribution in embryos of apterygote bristletail, Pedetontus unimaculatus (Fig. 5; Niwa et al., 2010). Thoracic lateral tergal regions between apterygote and pterygote insects are thus homologous, and some changes in homologous cells led to body wall extension.

Our functional analysis suggests that some changes in Wnt/Ft-Ds/Hippo pathways drove

277 dramatic growth of the homologous region to achieve powered flight. We showed critical roles of Wnt/Ft-Ds/Hippo pathways in the post-embryonic wing growth of Gryllus. Together with $v g$, these pathways comprise an FF signal circuit to expand the wing region in the 
281

282

283

284

285

286

287

288

289

290

291

292

293

294

295

296

297

298

299

300

301

302

303

304

hemimetabolous insect, exhibits repression of wing growth after post-embryonic depletion of $v g$ (Medved et al., 2015). These data suggest that the core wing growth mechanism is conserved between Gryllus and Drosophila (Fig. 5). Intriguingly, recent developmental studies revealed localized expression and function of $v g$, sd and $w g$ in crustacean flat epidermal outgrowths such as carapace, tergal edge and coxal plate, although homology of these flat outgrowths to insect wings remains controversial (Shiga et al., 2017; Clark-Hachtel and Tomoyasu, 2020; Bruce and Patel, 2020). Because these three genes are components of $v g$ FF signal, it suggests crustacean origin of this modular growth and either redeployment or reactivation in the lateral tergal cells extends the body wall of the wingless ancestor that led to insect wings.

\section{MATERIALS AND METHODS}

\section{Animal husbandry}

Gryllus bimaculatus strain used in this study derives from the gwhite strain (Niwa et al., 1997). Cricket colonies were reared in an air-conditioned room at $28-30{ }^{\circ} \mathrm{C}$ with $12 \mathrm{~L}: 12 \mathrm{D}$ photoperiod, and fed on artificial fish food (Spectrum Brands) and cat food (Purina One, Nestlé Purina Petcare). We kept nymphs for staging in an incubator (Panasonic) at $29{ }^{\circ} \mathrm{C}$ to minimize developmental stage variation at a time point. Embryo staging followed Donoughe and Extavour (2015).

\section{In situ hybridization}

We searched $v g$ and ap orthologs from Gryllus draft genome with BLAST and identified partial nucleotide sequences (vg: LC589559, apA: LC589561 and apB: LC589562; Ylla et al., 2021). The public sequence was used for $w g$ (AB044713.1). Digoxigenin-labeled riboprobes 
were transcribed in vitro from 188 to 523 bp DNA fragments amplified with primers that have until use.

310 The following procedures were performed at room temperature otherwise described. Fixed embryos were rehydrated in a $\mathrm{MeOH}$ series, digested in $2 \mu \mathrm{g} / \mathrm{ml}$ Proteinase $\mathrm{K}$ for $5 \mathrm{~min}$, and postfixed in $4 \%$ formaldehyde for 20 min. After prehybridization in hybridization buffer $(50 \%$ deionized formamide, 5X SSC, $100 \mu \mathrm{g} / \mathrm{ml}$ heparin, $100 \mu \mathrm{g} / \mathrm{ml}$ yeast RNA, $0.1 \%$ TritonX-100, of a riboprobe were hybridized with gentle shaking for more than 60 hours at $60-65{ }^{\circ} \mathrm{C}$. Hybridized embryos were washed with a series of SSC buffer at the hybridized temperature, then with maleic acid buffer, and then blocked in 1.5\% Roche blocking reagent (11096176001, Merck \& Co.) for more than an hour. Blocked embryos were incubated in 1:2,000 antiDigoxigenin-AP (11093274910, Merck \& Co.) overnight at $4^{\circ} \mathrm{C}$. After several washes with $1.5 \%$ Roche blocking reagent and maleic acid buffer, color was developed with NBT/BCIP solution.

\section{Size measurement}

We collected all samples from a single colony for minimizing variation from genetic and environmental background for assessment of area measurement in central and lateral regions in thoracic segments. We kept all hatched first instar nymphs in a plastic cage, and collect six or seven individuals after each molting. Each sex was separately collected from 
329

330

331

332

333

334

335

336

337

338

339

340

341

342

343

344

345

346

347

348

350

351

352

at $65^{\circ} \mathrm{C}$ after cutting off unnecessary body parts, and remaining tergal cuticles of thoracic segments were mounted on glass slides. We mounted nymph specimens in Hoyer's media. Adult specimens were washed in $100 \% \mathrm{EtOH}$ and mounted in EUKITT neo (O. Kindler ORSAtec).

Images of mounted slides were captured with a DFC7000 T equipped with a M165 FC (Leica Microsystemsy) and analyzed with ImageJ (version 2.0.0).

\section{CRISPR/Cas9-mediated genome editing}

We assembled oligonucleotides and transcribed and purified single-guide (sg) RNAs with a precision gRNA synthesis kit (A29377, ThermoFisher Scientific,) according to the manufacturer's instruction. Oligonucleotides used for DNA template assembly are shown in Table S4. Synthesized sgRNAs were aliquoted in small volumes and stored at $-80^{\circ} \mathrm{C}$ until use. For microinjection, eggs laid within 1-2 hours were collected from wet paper towels, and pieces of cotton layered in plastic dishes and were aligned in wells in a $2 \%$ agarose gel after a brief wash in tap water. A pulled glass capillary connected to a Femtojet microinjector (Eppendolf) was used to inject small droplets of solution into eggs. Materials were injected within 4 hours after egg oviposition.

For somatic gene knockouts, we injected $100 \mathrm{ng} / \mu \mathrm{l}$ sgRNA designed for each gene, and 500 $\mathrm{ng} / \mu \mathrm{l}$ Streptococcus pyogenes Cas9 protein (Integrated DNA Technologies). Nonhomologous end joining (NHEJ)-mediated gene knock-in is performed as previously described for generating the $v g$ reporter line (Watanabe et al., 2017). The donor plasmid was generated by integrating a partial DsRed sequence as sgRNA target and EGFP expression cassette driven by Gryllus cytoplasmic actin promoter (Zhang et al., 2002). A sgRNA targeting $5^{\prime}$ region of the $\mathrm{vg}$ protein-coding sequence was designed with a partial sequence obtained 
by BLAST search against the Gryllus genome assembly (accession no. LC589560). We injected a solution containing $40 \mathrm{ng} / \mu \mathrm{l}$ of sgRNAs targeting the $v g$ upstream site and the donor plasmid, $100 \mathrm{ng} / \mu \mathrm{l}$ of the donor plasmid, and $100 \mathrm{ng} / \mu \mathrm{l}$ of Cas9 mRNA transcribed from the linearized MLM3613 plasmid (Addgene 42251; Dahlem et al., 2012). GFP-positive individuals were crossed to wildtype, and individuals with GFP signal were selected for establishing the $v g$ reporter line.

\section{Tergum ablation}

361 The ablation was performed on third instar nymphs within 24 hours of molting. Nymphs were anesthetized on ice, and a targeted region of mesotergum on the right-hand side was ablated with a pair of spring scissors. The yellowish line is used as a landmark for ablating different sizes of tissue (i.e. LS and LL in Fig. 3A). Ablated nymphs were separately kept in a plastic cup until eclosion, and mesoterga of adults were digested with lactic acid and mounted on glass slides for area measurement. Cumming plots were created with DABEST (Ho et al., 2019).

\section{Quantitative PCR (qPCR)}

370 Nymphs newly molted within two hours to the third instar were periodically collected from a colony and separately kept in small plastic cups at a $29^{\circ} \mathrm{C}$. Three to four nymphs were moved on ice for anesthetization at each time point, and thoracic segments without an alimentary canal were dissected in ice-cold PBS, collected individually in TRIzol (Thermo Fisher Scientific), and stored at $-80{ }^{\circ} \mathrm{C}$ until use. Total RNA was extracted according to manufacturer's instructions, RNA pellets were resuspended in $30 \mu \mathrm{l}$ of distilled water and 

(Takara Bio) was used for qPCR reaction, and we calculated relative gene expression level with the delta Ct method.

382

mRNA-sequencing analysis

Nymphs molted within three hours to the third instar were periodically collected from a colony and separately kept in small plastic cups at a $29^{\circ} \mathrm{C}$ incubator. Zero and 3 DPE samples were anesthetized on ice within 10 min to 2.5 hours and 69 to 73 hours post ecdysis, respectively. Lateral and central tergal parts of both meso- and metathorax were dissected on a paper towel on ice, and then washed in PBS. Dissected tissues from 25 nymphs for a sample were collected in TRIzol (Thermo Fisher Scientific) and stored at $-80^{\circ} \mathrm{C}$ until use. Total RNA was extracted following the manufacturer's instructions and was further purified with a RNeasy MinElute spin column (QIAGEN). We also extracted total RNA from embryos incubated at $29^{\circ} \mathrm{C}$ at days $2.5,3,3.5,4.5,5.5$ and 6 after egg laying. Total RNA from terga and embryos were eluted from a column with $20 \mu \mathrm{l}$ of RNase-free water and submitted to GENEWIZ and Filgen standard RNA-seq service (GENEWIZ) that provided a standard Illumina mRNA library and generated 150 bp pair-end reads on an Illumina HiSeqX and HiSeq4000 (Illumina), respectively. Raw reads were deposited to DDBJ/EBI/NCBI database (PRJDB10701). 
401

402

403

404

405

406

407

408

409

410

411

412

413

414

415

416

417

418

419

420

421

422

423

424

of the expression level of each transcript with Salmon (v1.0.1; Patro et al., 2017), DEGs were statistically identified with DESeq2 (v1.26.0; Love et al., 2014). Functional annotation of assembled transcripts was performed with both Trinotate (v3.1.0) and BLASTX (v2.9.0) against the latest Drosophila protein sequences (r6.32). The FlyBase ID of the best BLAST hits in the search against Drosophila database was assigned to each transcript and used for gene set analysis with DAVID (v6.8; Dennis et al., 2003). Data were visualized with EnhancedVolcano (v1.7.10; Blighe et al., 2020), Python3 (v3.7.7) and R (v3.6.3).

\section{Nymphal RNAi}

For the functional screening, we used a consensus sequence created from all isoforms for a target gene in the transcriptome to design a double-stranded RNA (dsRNA). The specificity of dsRNA was assessed by BLAST search to both the transcriptome assembled in this study and the genome (Ylla et al., 2021).

RNA was transcribed in vitro from a PCR product as a template. PCR primers are listed in Table S4. Transcribed RNA was digested with DNase I, then purified with a standard phenol/chloroform extraction protocol. After annealing, dsRNA was aliquoted and stored at $80{ }^{\circ} \mathrm{C}$ until use. One $\mu \mathrm{l}$ of dsRNA was injected into the ventral side of the intersegmental membrane between second and third thoracic segments with a pulled glass capillary. Nymphs injected with the same dsRNA were kept in a plastic cage until reaching adulthood.

\section{Image analysis}

Epifluorescent/Confocal/scanning electron microscopy images were taken with an M165 FC (Leica microsystems)/A1R MP (Nikon)/VHX-D500 (KEYENCE), respectively. Images were processed with either ImageJ (version 2.0.0) or GIMP (version 2.10) and assembled and 
annotated with Inkscape (version 1.0 beta).

426

\section{Acknowledgements}

428 We thank Drs. Tetsuya Bando and Sumihare Noji for their help in preliminary analysis of 429 Gryllus vg and sd function. We thank Dr. Toshiya Ando, and Dr. Takaaki Daimon and his lab 430 members for helpful discussion. We also thank Drs. Yuji Matsuoka, Takahito Watanabe, 431 Yohei Katoh, Taro Nakamura, Shinichi Morita, Hajime Ono, Miki Sugimoto, and Mr. Takahisa 432 Yamashita for technical supports. The computational resource for the RNA-seq analysis was 433 provided by NIG supercomputer system. This study was supported by MEXT KAKENHI 434 (16K18825 and $19 \mathrm{H} 02970$ for TO, $16 \mathrm{H} 02596$ for TN).

435 


\section{REFERENCES}

437 Ashburner, M. \& Richards, G. Sequential gene activation by ecdysone in polytene chromosomes of Drosophila melanogaster. III. Consequences of ecdysone withdrawal. Dev. Biol. 54, 241-255 (1976).

Averof, M. \& Cohen, S. M. Evolutionary origin of insect wings from ancestral gills. Nature 385, $627-630$ (1997).

Bernard, F. et al. Control of apterous by vestigial drives indirect flight muscle development in Drosophila. Dev. Biol. 260, 391-403 (2003).

Blighe, K., Rana, S. \& Lewis, M. EnhancedVolcano: Publication-ready volcano plots with enhanced colouring and labeling. $\mathrm{R}$ package version 1.7.10, https://github.com/kevinblighe/EnhancedVolcano (2020).

Bruce, H. S. \& Patel, N. H. Knockout of crustacean leg patterning genes suggests that insect wings and body walls evolved from ancient leg segments Heather. Nat. Ecol. Evol. 4, 1703-1712 (2020).

Bruce, H. S. The Daphnia carapace and the origin of novel structures. Preprint at Preprints. https://doi.org/10.20944/preprints202102.0221.v1 (2021).

Clark-Hachtel, C. M., Linz, D. M. \& Tomoyasu, Y. Insights into insect wing origin provided by functional analysis of vestigial in the red flour beetle, Tribolium castaneum. Proc. Natl. Acad. Sci. U. S. A. 110, 16951-16956 (2013).

Clark-Hachtel, C. M. \& Tomoyasu, Y. Exploring the origin of insect wings from an evo-devo perspective. Curr. Opin. Insect Sci. 13, 77-85 (2016). and their implication for the origin of insect wings. Nat. Ecol. Evol. (2020).

Clark-Hachtel, C., Fernandez-Nicolas, A., Belles, X. \& Tomoyasu, Y. Tergal and pleural wing- 

origin of insect wings. Evol. Dev. e12372 (2021).

Crampton, G. The phylogenetic origin and the nature of the wings of insects according to the paranotal theory. J. New York Entomol. Soc. 24, 1-39 (1916).

Dabour, N. et al. Cricket body size is altered by systemic RNAi against insulin signaling components and epidermal growth factor receptor. Dev. Growth Differ. 53, 857-869 (2011).

Dahlem, T. J. et al. Simple methods for generating and detecting locus-specific mutations induced with TALENs in the zebrafish genome. PLoS Genet. 8, e1002861 (2012).

Dennis, G. Jr. et al. DAVID: Database for Annotation, Visualization, and Integrated Discovery. Genome Biol. 4, P3 (2003).

Diaz-benjumea, F. J. \& Cohen, S. M. Interaction between dorsal and ventral cells in the imaginal disc directs wing development in Drosophila. Cell 75, 741-752 (1993).

Diaz-Benjumea, F. J. \& Cohen, S. M. Serrate signals through Notch to establish a Winglessdependent organizer at the dorsal/ventral compartment boundary of the Drosophila wing. Development 121, 4215-4225 (1995).

Donoughe, S. \& Extavour, C. G. Embryonic development of the cricket Gryllus bimaculatus. Dev. Biol. 411, 1-16 (2015).

Furukawa, N., Tomioka, K. \& Yamaguchi, T. Functional anatomy of the musculature and 480 innervation of the neck and thorax in the cricket, Gryllus bimaculatus. Zool. Mag. 92, 371-385 (1983).

Grabherr, M. G. et al. Full-length transcriptome assembly from RNA-seq data without a reference genome. Nat. Biotechnol. 29, 644-652 (2011).

Hamaratoglu, F., Affolter, M. \& Pyrowolakis, G. Dpp/BMP signaling in flies: From molecules 

analysis with estimation graphics. Nat. Methods 16, 565-566 (2019). 366, 1004-1007 (2019).

Kim, J., Irvine, K. D. \& Carroll, S. B. Cell recognition, signal induction, and symmetrical gene activation at the dorsal-ventral boundary of the developing Drosophila wing. Cell 82, 795-802 (1995).

Kim J. et al. Integration of positional signals and regulation of wing formation and identity by Drosophila vestigial gene. Nature 382, 133-138 (1996). Can. J. Zool. 61, 1618-1669 (1983).

Kukalová-Peck, J. Phylogeny of higher taxa in Insecta: Finding synapomorphies in the extant fauna and separating them from homoplasies. Evol. Biol. 35, 4-51 (2008).

Li, T., White, K. P. \& Haven, N. Tissue-specific gene expression and ecdysone-regulated genomic networks in Drosophila. Dev. Cell 5, 59-72 (2003).

Linz, D. M. \& Tomoyasu, Y. A dual evolutionary origin of insect wings supported by an investigation of the abdominal wing serial homologs in Tribolium. Proc. Natl. Acad. Sci. U. S. A. 9, E658-E667 (2018).

Love, M. I., Huber, W. \& Anders, S. Moderated estimation of fold change and dispersion for RNA-seq data with DESeq2. Genome Biol. 15, 550 (2014). reads. EMBnet.journal 17, 10-12 (2011). 
origin of pleuron in insects. Sci. Rep. 7, 12597 (2017).

Medved, V. et al. Origin and diversification of wings: Insights from a neopteran insect. Proc. Natl. Acad. Sci. 112, 15946-15951 (2015).

Miyawaki, K. et al. Involvement of Wingless/Armadillo signaling in the posterior sequential segmentation in the cricket, Gryllus bimaculatus (Orthoptera), as revealed by RNAi analysis. Mech. Dev. 121, 119-130 (2004).

Nijhout, H. F. Insect Hormones. (Princeton University Press, 1994).

Nijhout, H. F. \& Grunert, L. W. Bombyxin is a growth factor for wing imaginal disks in Lepidoptera. Proc. Natl. Acad. Sci. U. S. A. 99, 15446-15450 (2002).

Nijhout, H. F., Laub, E. \& Grunert, L. W. Hormonal control of growth in the wing imaginal disks of Junonia coenia: the relative contributions of insulin and ecdysone. Development

Niwa, N., Saitoh, M., Ohuchi, H., Yoshioka, H. \& Noji, S. Correlation between Distal-less expression patterns and structures of appendages in development of the two-spotted cricket, Gryllus bimaculatus . Zool. Sci. 14, 115-125 (1997).

Niwa, N. et al. Evolutionary origin of the insect wing via integration of two developmental modules. Evol. Dev. 12, 168-176 (2010).

Ohde, T., Yaginuma, T. \& Niimi, T. Insect morphological diversification through the modification of wing serial homologs. Science 340, 495-498 (2013). bias-aware quantification of transcript expression. Nat. Methods 14, 417-419 (2017). 
532

533

534

535

536

537

538

539

540

541

542

543

544

545

546

547

548

549

550

551

552

553

554

555

Prokop, J. et al. Paleozoic nymphal wing pads support dual model of insect wing origins. Curr. Biol. 27, 263-269 (2017).

R Core Team. R: A language and environment for statistical computing. R Foundation for Statistical Computing, https://www.R-project.org/ (2020).

Rasnitsyn, A. P. A modified paranotal theory of insect wing origin. J. Morphol. 168, 331-338 (1981).

Schaffner, K. H., \& Koch, U. T. A new field of wing campaniform sensilla essential for the production of the attractive calling song in crickets. J. Exp. Biol. 129, 1-23 (1987).

Seppey M., Manni M. \& Zdobnov E. M. BUSCO: Assessing genome assembly and annotation completeness. In: Kollmar M. (eds) Gene Prediction. Methods in Molecular Biology, vol 1962. (Humana Press, 2019).

Shiga, Y. et al. Repeated co-option of a conserved gene regulatory module underpins the evolution of the crustacean carapace, insect wings and other flat outgrowths. Preprint at bioRxiv https://doi.org/10.1101/160010 (2017).

Snodgrass, R. E. Principles of Insect Morphology. (McGraw-Hill Book Company, 1935).

Sudarsan, V., Anant, S., Guptan, P., Vijayraghavan, K. \& Skaer, H. Myoblast diversification and ectodermal signaling in Drosophila. Dev. Cell 1, 829-839 (2001).

Tiegs, O. W. The flight muscles of insects-their anatomy and histology; with some observations on the structure of striated muscle in general. Philos. Trans. R. Soc. B Biol. Sci. 238, 221-348 (1955).

Tomoyasu, Y. What crustaceans can tell us about the evolution of insect wings and other morphologically novel structures. Curr. Opin. in Genet. Dev. 69, 48-55 (2021).

Truman, J. W. \& Riddiford, L. M. The evolution of insect metamorphosis. Philos. Trans. R. Soc. B 29, R1252-R1268 (2019). 
556 Watanabe, T., Noji, S. \& Mito, T. Genome editing in the cricket, Gryllus bimaculatus. In: Hatada I. (eds) Genome Editing in Animals. Methods in Molecular Biology, vol 1630. (Humana Press, 2017).

Wigglesworth, V. Evolution of insect wings and flight. Nature 246, 127-129 (1973). by the nuclear vestigial gene product. Genes Dev. 5, 2481-2495 (1991).

Williams, J. A., Paddock, S. W., Vorwerk, K. \& Carroll, S. B. Organization of wing formation and induction of a wing-patterning gene at the dorsal/ventral compartment boundary. Nature 368, 299-305 (1994).

Ylla, G. et al. Insights into the genomic evolution of insects from cricket genomes. Preprint at bioRxiv https://doi.org/10.1101/2020.07.07.191841 (2021).

Zecca, M. \& Struhl, G. Control of Drosophila wing growth by the vestigial quadrant enhancer. Development 134, 3011-3020 (2007).

Zecca, M. \& Struhl, G. A feed-forward circuit linking Wingless, Fat-Dachsous signaling, and the Warts-Hippo pathway to Drosophila wing growth. PLoS Biol. 8, e1000386 (2010). embryos of the cricket Gryllus bimaculatus. Dev. Growth Differ. 44, 409-417 (2002). 

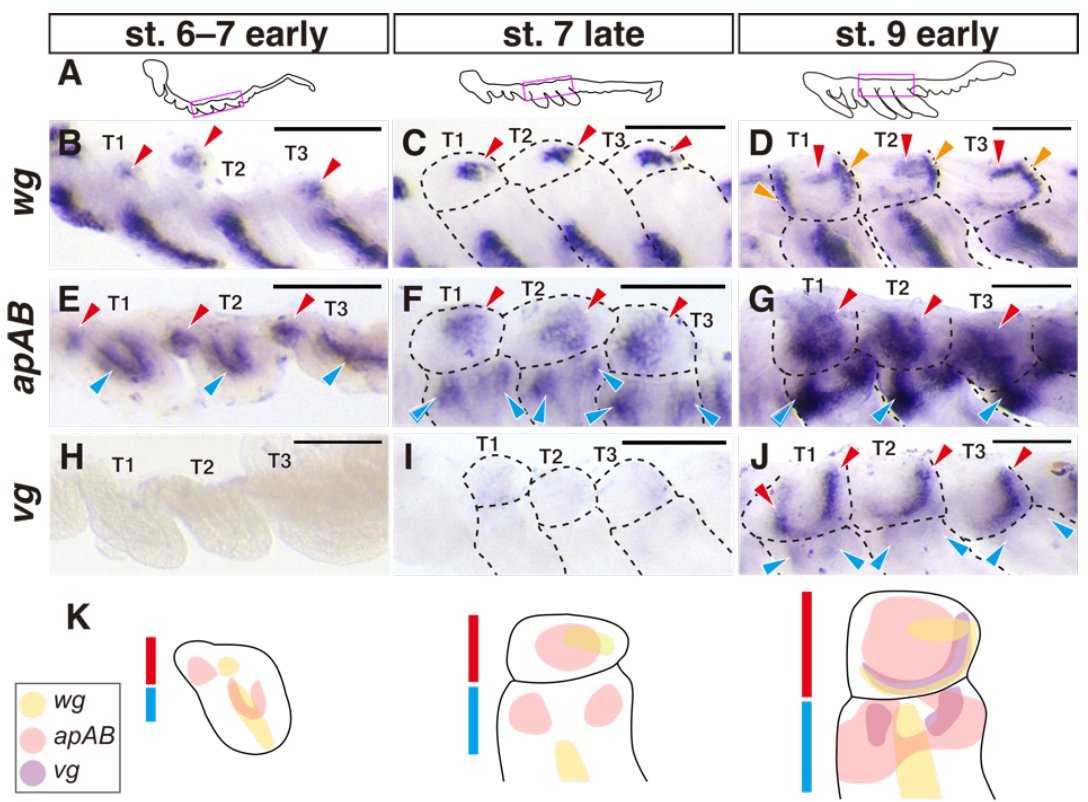

574

575

576

577

578

579

580

581

582

583

584

585

586

587
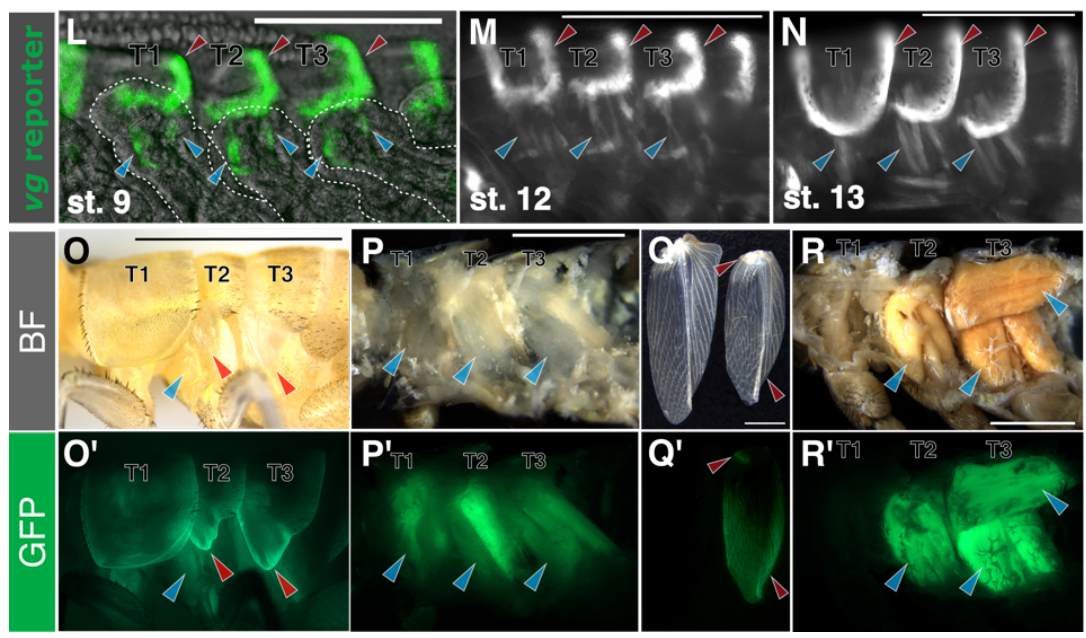

Fig. 1 Expression pattern of $w g, a p A B$ and $v g$ in thoracic segments of Gryllus. A,

Schematics illustrate lateral views of Gryllus embryos in each stage. Magenta rectangle indicates the region shown in B-J. B-J Dorsolateral regions of thoracic segments stained for $w g(\mathbf{B}-\mathbf{D})$, $\operatorname{ap} A B(\mathbf{E}-\mathbf{G})$ and $v g(\mathbf{H}-\mathbf{J})$ with in situ hybridization in stage 6 to early stage $7(\mathbf{B}, \mathbf{E}, \mathbf{H})$, late-stage 7 (C, F, I) and early-stage 9 (D, G, J). K, Summary of gene expression pattern in meso- and metathoracic segments. Red and blue lines indicate tergal and pleural regions, respectively. L-N $v g$ reporter gene signals in dorsolateral regions of thoracic segments in late embryos. $\mathbf{O}-\mathbf{R}$ and O'-R' vg reporter gene signals in post-embryonic stages. Dorsolateral region $\left(\mathbf{O}, \mathbf{O}^{\prime}\right)$ and thoracic musculature in median section (P, $\left.\mathbf{P}^{\prime}\right)$ of sixth instar nymph, and forewing $\left(\mathbf{Q}, \mathbf{Q}^{\prime}\right)$ and thoracic musculature in median section ( $\mathbf{R}, \mathbf{R}^{\prime}$ ) of adult. Crickets before cuticle coloration are shown for both nymph and adult. Red and blue arrowheads indicate expression in tergal and pleural regions, respectively. Scale bars are $100 \mu \mathrm{m}$ in B-J, $250 \mu \mathrm{m}$ in $\mathbf{L}-\mathbf{N}$ and $3 \mathrm{~mm}$ in $\mathbf{0}-\mathbf{R}$, respectively. 


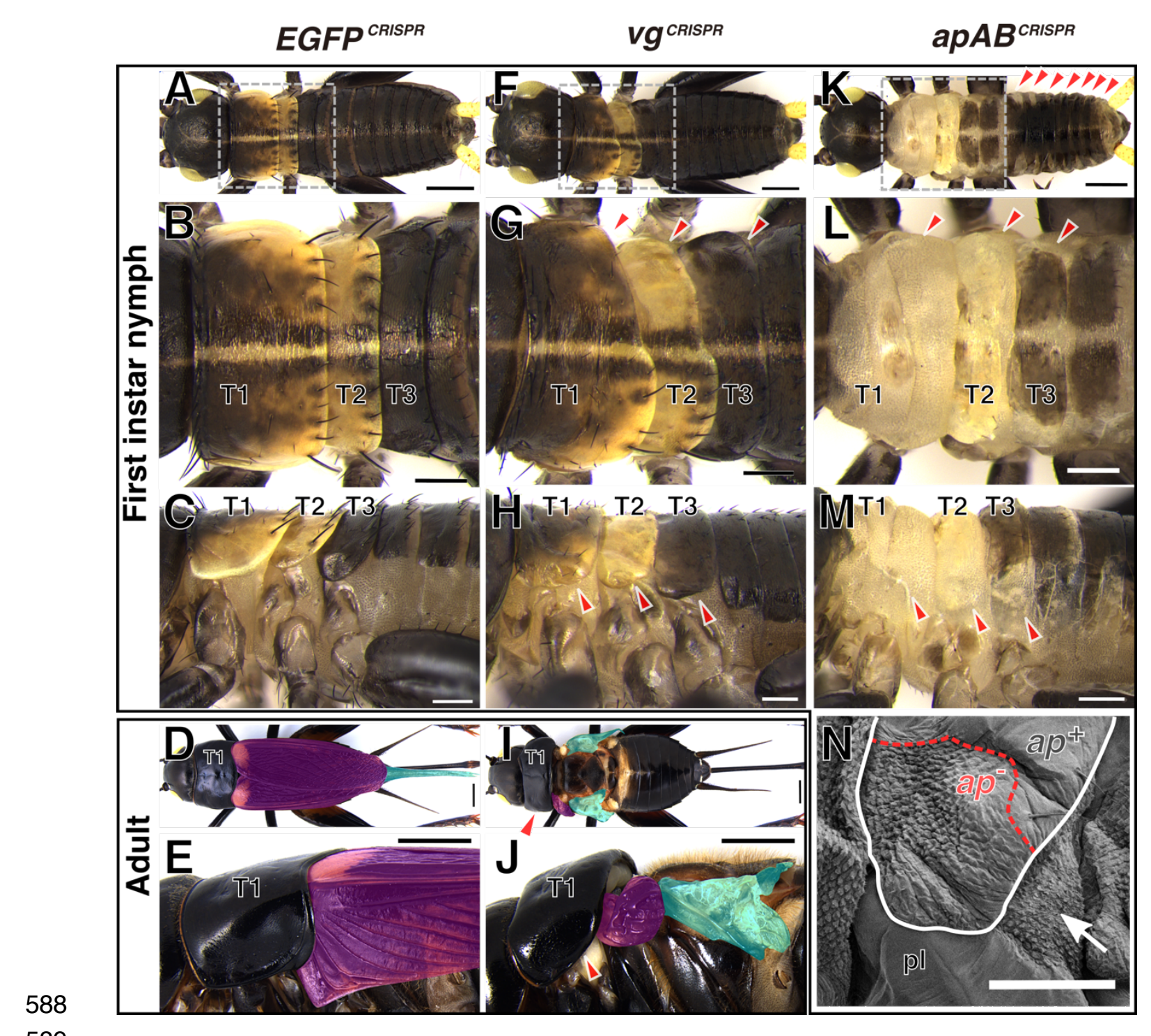

590 Fig. 2 Mosaic knockouts of $\mathbf{v g}$ and apAB. A-M, Representative images of first instar nymphs 591 (A-C, F-H, K-M) and female adults (D, E, I, J) injected with Cas9 protein and sgRNAs targeting $592 \operatorname{EGFP}(\mathbf{A}-\mathbf{E}), v g(\mathbf{F}-\mathbf{J})$ and $\operatorname{apAB}(\mathbf{K}-\mathbf{M})$ in early embryos from dorsal $(\mathbf{A}, \mathbf{B}, \mathbf{D}, \mathbf{F}, \mathbf{G}, \mathbf{I}, \mathbf{K}, \mathbf{L})$ and lateral $(\mathbf{C}, \mathbf{E}, \mathbf{H}, \mathbf{J}, \mathbf{M})$ views. Boxed area in $\mathbf{A}, \mathbf{F}, \mathbf{K}$ is magnified in $\mathbf{B}, \mathbf{G}$, $\mathbf{L}$, respectively. Arrowheads indicate regions affected by gene knockouts. Fore- and hindwings are shaded in magenta and cyan, respectively. Arrowheads indicate affected tergal regions. n A scanning electron micrograph of the dorsolateral region in mesothorax of an apAB mosaic knockout cricket. The tergum is outlined with a white line. Tergal surface with specific $(a p)$ and wildtype $\left(a p^{+}\right)$ phenotypes separated by red dotted line. The rough surface structure of ap area resembles a soft intersegmental region (arrow). pl, pleuron. Scale bars are $250 \mu \mathrm{m}$ in $\mathbf{A}-\mathbf{C}, \mathbf{F}-\mathbf{H}, \mathbf{K}-\mathbf{M}, 2 \mathrm{~mm}$, D, E, I, J and $100 \mu \mathrm{m}$ in $\mathbf{N}$. 


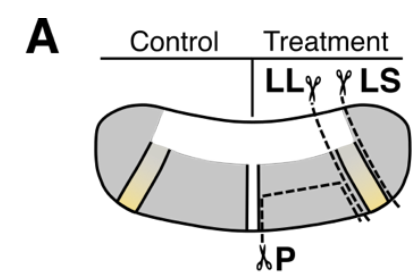

B
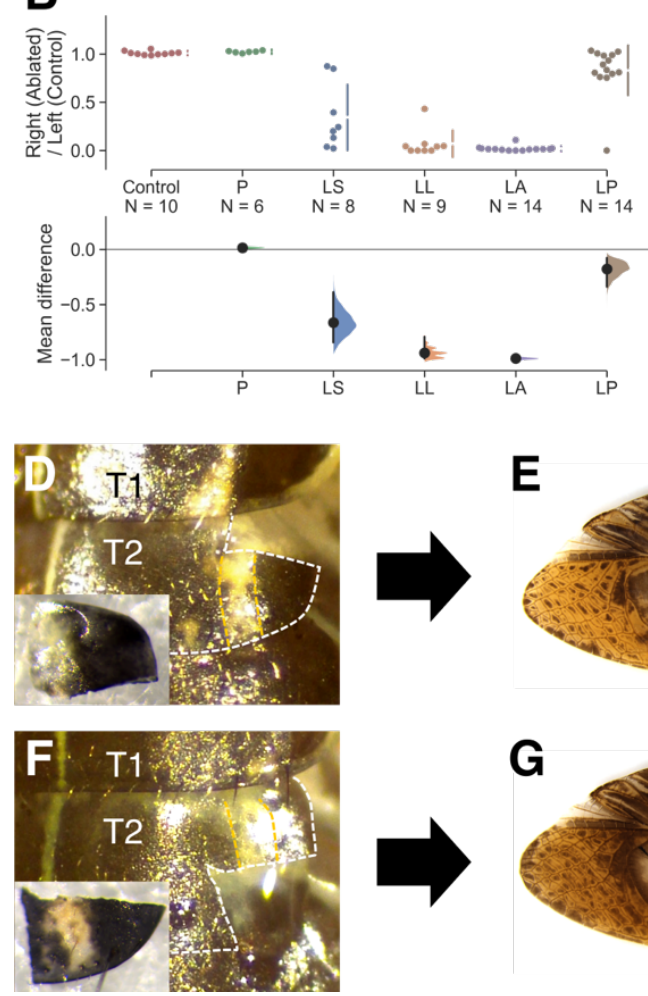

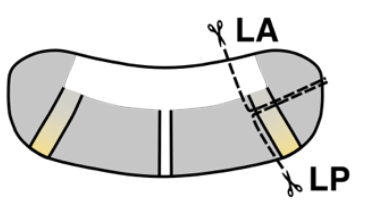

C

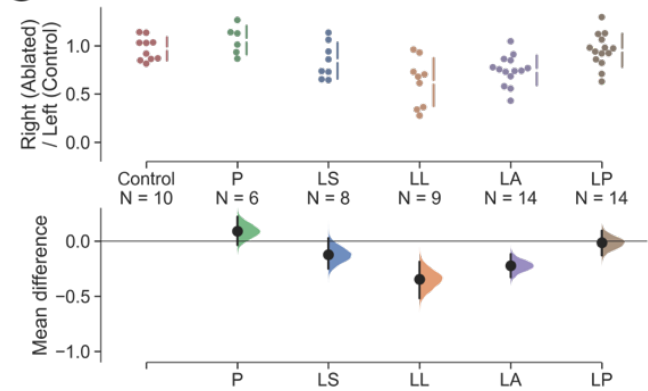

602

603

604

605

606

607

608

609

610

611

612

613

614
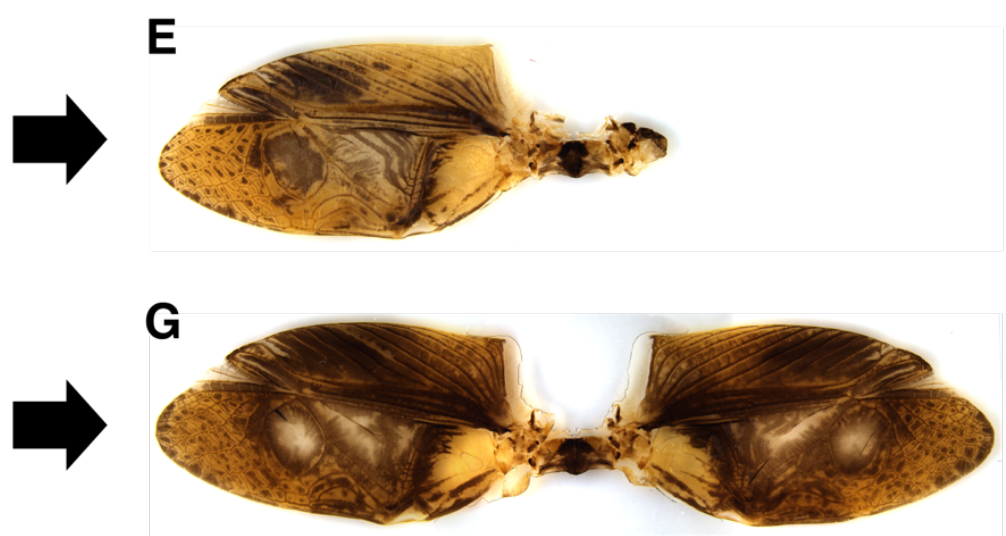

\section{Fig. 3 Identification of wing growth organizer in the lateral-anterior region of thoracic terga.}

A, Schematic illustration indicates ablated regions in the third instar nymph: P, posterior; LS, lateral-small; LL, lateral-large; LA, lateral-anterior; LP, lateral-posterior. B, C, Effect of nymphal tergal ablations on wing $(\mathbf{B})$ and articulation $(\mathbf{C})$ sizes in adults. Cumming plots indicate relative area of the treated right side to untreated left side (top) and mean differences of the values from control for wing (B) and articulation (C) regions (bottom). D-G, Representative results of the ablation treatment. The right side of targa of the third instar nymph after the ablation of the LA (D) and LP (E) regions. The ablated tissues are shown in insets. Resultant adult wings of the same individuals are shown in F and $\mathbf{G}$, respectively. Mesoterga and the yellowish line is outlined with white and yellow dotted lines, respectively. 


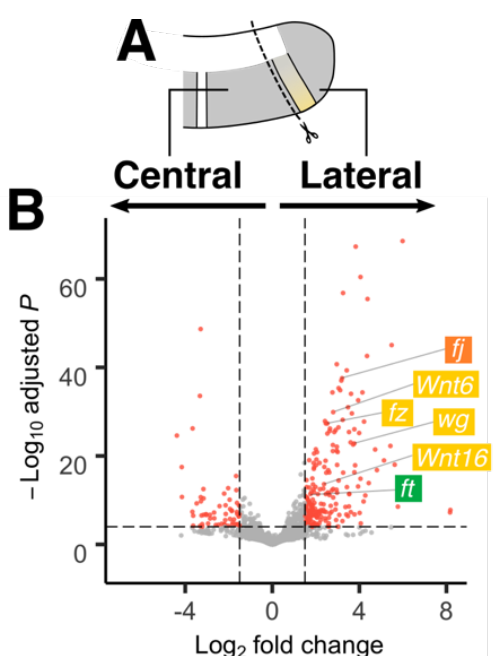

C

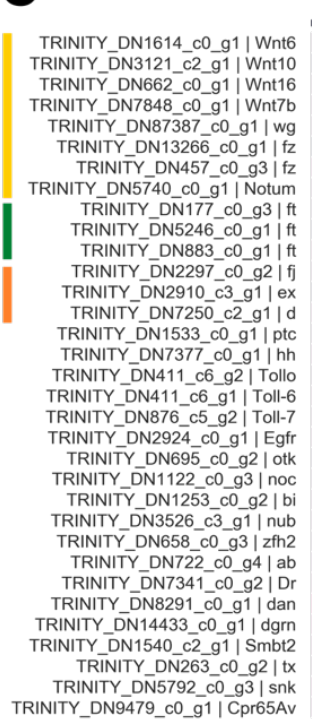

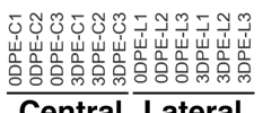

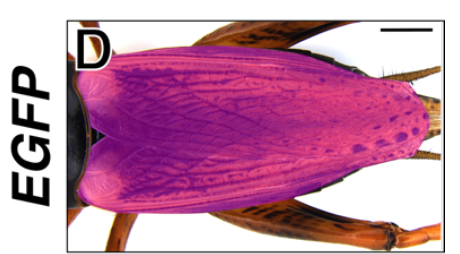
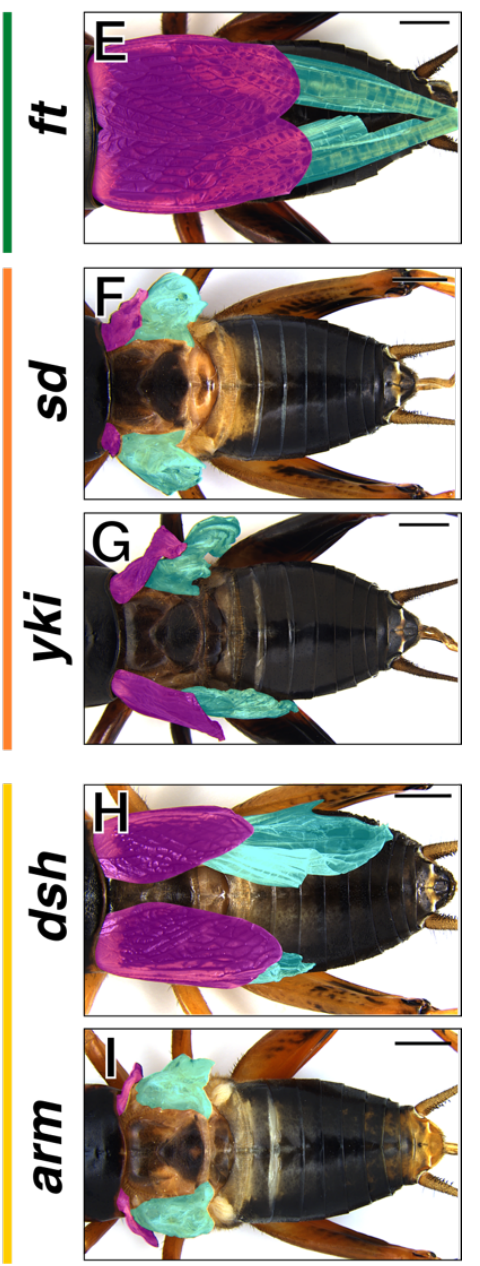

616 Fig. 4 Wnt, Ft-Ds and Hippo signaling are required for the post-embryonic wing growth in

617 Gryllus. A, Schematics illustrates tergal regions used for the transcriptome comparison. Note

618 that only T2 and T3 segments were used for this analysis. B, A volcano plot shows differential 619 expression of transcripts between central and lateral regions in T2 and T3 terga. Data after log620 fold change shrinkage with the apeglm method is visualized. Significantly differentially expressed 621 transcripts (adjusted $P<10 \mathrm{e}-5$, fold change $>1.5$ ) are in red. Some representative transcripts that are highly expressed in the lateral region are labeled. C, Heatmap indicating expression level of 33 candidate genes in each sample. Color code indicates z-score normalized transcripts per million (TPM). D-K, Adult phenotypes after nymphal RNAi treatment. Fore- and hindwings are shaded in magenta and cyan, respectively. Scale bars are $2 \mathrm{~mm}$. 


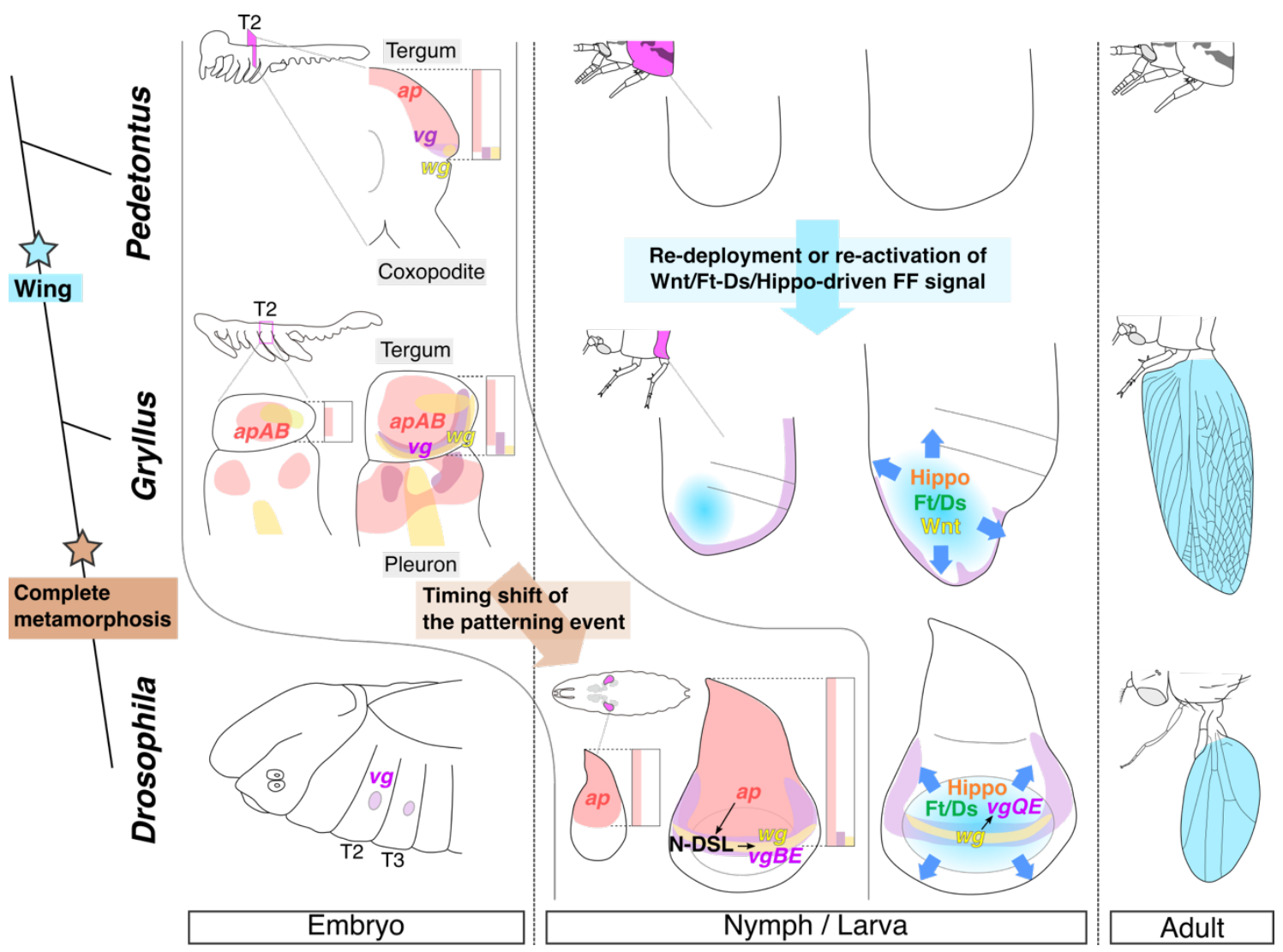

Fig. 5 The development and evolution of the wing blade from the tergal margin region.

630 A resemblance of the dorsoventral distribution of wg/ap/ $\mathrm{vg}$ between Pedetontus (Niwa et al., 631 2010) and Gryllus indicates that nymphal terga are patterned in a similar fashion. The similar 632 distribution is known in the Drosophila wing disc at the late second instar (Kim et al., 1995), which 633 implicates a timing shift of the patterning event from embryonic to larval stage. vgBE activity in 634 Drosophila is required to initiate expansion of the wing compartment via $v g$ FF signaling 635 transduced through actions of vgQE, Wg, and Ft-Ds and Hippo pathways (Zecca and Struhl, 636 2010). Analogously, the vg-dependent tergal margin in Gryllus is required for the wing growth, 637 and Wnt, Ft-Ds and Hippo pathways drive dramatic expansion during post-embryonic 638 development. A growth mechanism like that in Drosophila was employed at the lateral tergal 639 region of an ancestral apterygote insect to form the body wall extension required for the evolution 640 of insect flight. 